\title{
DENSIDAD DEL ACEITE SHELL HELIX HX 10W-40, EN FUNCIÓN DE LA TEMPERATURA
}

\author{
SHELL HELIX HX7 10W-40 OIL DENSITY, DEPENDING ON THE TEMPERATURE
}

\author{
Carlos Job Fiestas Urbina1', Benigno Benito Lizárraga Zavaleta²
}

\begin{abstract}
RESUMEN
Se ha medido la densidad en función de la temperatura del aceite Shell Helix HX7 10W-40, con el objetivo de utilizarlo como líquido manométrico de un micro manómetro diferencial de un tubo de Pitot destinado a medir la velocidad del aire de un sistema de medición de fuerzas de arrastre de perfiles aerodinámicos. El aceite se colocó en una probeta de laboratorio graduada de vidrio de $500(\mathrm{ml})$, se calentó en baño de maría utilizando una hornilla eléctrica de $2500(\mathrm{~W})$ y un depósito cilíndrico de aluminio, hasta alcanzar una temperatura de $48.5\left({ }^{\circ} \mathrm{C}\right)$ en el nivel 250 (ml). Se dejó enfriar al medio ambiente y se tomaron varias mediciones de su densidad conforme iba enfriando a diferentes temperaturas del aceite. El resultado de esta medición fue una correlación empírica de la densidad del aceite expresada en $\left(\mathrm{Kg} / \mathrm{m}^{3}\right)$ como función de la temperatura expresada en $\left({ }^{\circ} \mathrm{C}\right)$. Esta correlación posee un coeficiente de correlación $\mathrm{R}^{2}=0.991766$ y predice una densidad $\rho=875.20\left(\mathrm{Kg} / \mathrm{m}^{3}\right)$ a la temperatura T $=4\left({ }^{\circ} \mathrm{C}\right)=277.15(\mathrm{~K})$

Palabras clave: Aceite lubricante; Densidad; temperatura.
\end{abstract}

\begin{abstract}
The density has been measured according to the temperature of the Shell Helix HX7 10W-40 oil, with the objective of using it as a manometric liquid of a differential micro gauge of a Pitot tube for measuring the air velocity of a measuring system for drag forces of aerodynamic profiles. The oil was placed in a glass graduated laboratory test tube of $500(\mathrm{ml})$, heated in a bain-marie using a $2500(\mathrm{~W})$ electric cooker and an aluminum cylindrical tank, until a temperature of $48.5\left({ }^{\circ} \mathrm{C}\right)$ was reached at $250(\mathrm{ml})$ level. It was allowed to cool to the environment and several measurements of its density were taken as it was cooling at different oil temperatures. The result of this measurement was an empirical correlation of oil density expressed in $\left(\mathrm{Kg} / \mathrm{m}^{3}\right)$ as a function of the temperature expressed in $\left({ }^{\circ} \mathrm{C}\right)$. This correlation has a correlation coefficient $\mathrm{R}^{2}=0.991766$ and predicts a density $\rho=875.25\left(\mathrm{Kg} / \mathrm{m}^{3}\right)$ at the temperature $\mathrm{T}=4\left({ }^{\circ} \mathrm{C}\right)=277.15(\mathrm{~K})$
\end{abstract}

Keyword: Lubricating oil; Density; temperature.

\footnotetext{
${ }_{1}^{1}$ Docente. Facultad de Ciencias. Universidad Nacional José Faustino Sánchez Carrión. Lima - Perú Email: carfieur@yahoo.es

${ }^{2}$ Docente. Facultad de Ingeniería Química y Metalúrgica. Universidad Nacional José Faustino Sánchez Carrión. Lima - Perú Email: benitolizarraga@gmail.com
} 


\section{INTRODUCCIÓN}

Para medir la velocidad del aire entre 0.5 a $3(\mathrm{~m} / \mathrm{s})$ es necesario utilizar tubos de Pitot de alta sensibilidad, en los cuales se utiliza como líquido manométrico aceites o alcoholes de baja densidad.

Con el fin de adaptar un tubo de Pitot mara medir bajas velocidades del aire se ha seleccionado como líquido manométrico al aceite lubricante Shell Helix HX7 10W-40, por su baja densidad y su definida coloración.

Para utilizar eficientemente este aceite como líquido manométrico, se ha desarrollado un experimento para medir su densidad en función de la temperatura del aceite, que es el motivo del presente artículo.

\section{FORMULACIÓN DEL PROBLEMA}

Para definir el problema principal, se requiere responder las siguientes preguntas.

- ¿Será posible medir la densidad del aceite lubricante Shell Helix HX7 10W-40, en función de la temperatura?

- ¿Será posible obtener una correlación empírica entre la densidad y la temperatura del aceite Shell Helix HX7 10W-40?

\section{ANTECEDENTES}

-Título: Efecto de la temperatura sobre la densidad de biodiesel de aceite de palma y sus mezclas con diesel convencional. Revista energética (Diciembre del 2006). Universidad Nacional de Colombia.

Autores: Benjumea, P., Chávez, G., Vargas, C.

Resumen: En este artículo se presentan los fundamentos teóricos y resultados experimentales de la densidad del biodiesel de aceite crudo de palma y sus mezclas en función de su temperatura.

-Título: Dilatación térmica.

Autor: Wikipedia (10 de Diciembre del 2017). Coeficiente de dilatación térmica. Disponible en: https://es.wikipedia.org/wiki/Coeficiente_de_dilataci $\%$ C3\%B3n.

Resumen: Se presenta el fundamento de la dilatación térmica de sólidos y líquidos, y tablas conteniendo el coeficiente de dilatación térmica de algunos sólidos y líquidos entre $0-100\left({ }^{\circ} \mathrm{C}\right)$.

\section{MARCOTEÓRICO}

Medición de la densidad de líquidos usando el densímetro o areómetro

La densidad de los líquidos se mide con mucha facilidad usando el densímetro o areómetro, el cual es un instrumento cuyo funcionamiento se basa el principio de Arquímedes.

Figura 1. Densímetro o areómetro

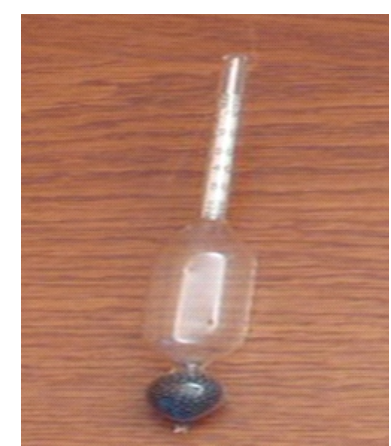

Fuente: Wikipedia

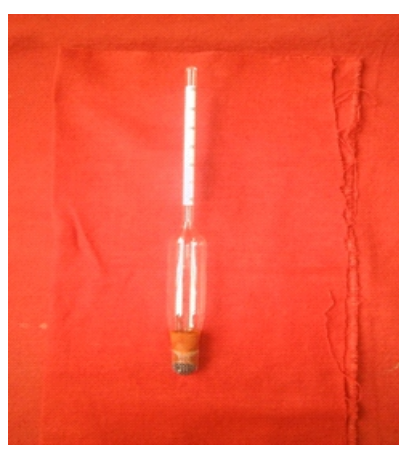

Fuente: Fotografía propia
Es un tubo cerrado con un bulbo pesado en uno de sus extremos y en forma de varilla delgada en el otro extremo. En el interior de la varilla delgada está colocada una escala que indica la densidad relativa del líquido cuya densidad se mide.

Se introduce el bulbo en el líquido que es evaluado y el densímetro flota de modo tal que el nivel de la superficie libre del líquido indica sobre la escala graduada la densidad relativa del líquido.

\section{Medición de la densidad a diferentes temperaturas del líquido}

Cuando el líquido se encuentra temperaturas diferentes a la temperatura de calibración del areómetro, es necesario realizar correcciones.

El areómetro posee un peso y un volumen que varía según la temperatura del líquido que está midiendo.

El peso del areómetro es equilibrado por la fuerza de empuje hidrostático del agua y del líquido, cumpliéndose las siguientes expresiones.

Si realizamos la medición a la temperatura de calibración:

Durante la calibración, el densímetro se sumerge en agua destilada a la temperatura de calibración, resultando.

$$
P=\rho_{a g}(T c) V_{o}(T c) g
$$

Si sumergimos el densímetro en el líquido de interés a una temperatura $\mathrm{T}$, diferente de la temperatura de calibración. se obtiene la expresión

$$
P=\rho_{\text {Liq }}(T)\left[V_{0}(T)-V_{\text {ext }}(T)\right] g
$$

Igualando los segundos miembros de las ecuaciones (1) y (2), despejamos la densidad del líquido.

$$
\rho_{L}(T)=\frac{\rho_{a g}(T c) V_{o}(T c)}{\left[V_{0}(T)-V_{e x t}(T)\right]}
$$

Sin embargo, cuando se sumerge el densímetro a la temperatura $\mathrm{T}$, diferente a la temperatura de calibración, el volumen del areómetro varía por efecto de la dilatación térmica, distorsionando la medición de la densidad del líquido, si expresamos este efecto en la ecuación (3), obtenemos. 


$$
\rho_{L}(T)=\frac{\rho_{a g}(T c) V_{o}(T c)}{\left[V_{0}(T c)-V_{e x t}(T c)\right]\left[1+\alpha_{v}(T-T c)\right]}
$$

La densidad medida es dada por la expresion.

$$
\rho_{\text {medidaL }}(T)=\frac{\rho_{a g}(T c) V_{o}(T c)}{\left[V_{0}(T c)-V_{\text {ext }}(T c)\right]}
$$

Combinando las expresiones (4) y (5), obtenemos.

$$
\rho L_{\text {corr }}(T)=\frac{\rho L_{\text {medida }}(T)}{1+\alpha v_{\text {vidrio }}(T-T C)}
$$

\section{Donde,}

$\rho L_{\text {corr }}(T)$ : densidad del líquido corregida por el efecto de expansión térmica, $\left(\mathrm{Kg} / \mathrm{m}^{3}\right)$.

$\rho L m e d i d a(T)$ : densidad del líquido medida (indicada en el areómetro), $\left(\mathrm{Kg} / \mathrm{m}^{3}\right)$.

$\alpha v_{\text {vidrio }}$ : coeficiente volumétrico de expansión térmica del vidrio Pyrex, $\left({ }^{\circ} \mathrm{C}^{-1}\right)$.

Tc: temperatura de calibración del areómetro, $\left({ }^{\circ} \mathrm{C}\right)$.

T: temperatura de medición, $\left({ }^{\circ} \mathrm{C}\right)$.

\section{Expansión térmica del vidrio}

Las sustancias al calentarse aumentan la energía cinética lineal y vibratoria de las moléculas que las constituyen, esto hace que cada molécula incremente el volumen que ocupa y por lo tanto el cuerpo se dilata.

Las variaciones lineales de expansión térmica se representan por la expresión.

$$
L(T)=L(T o)\left[1+\alpha_{L}(T-T o)\right]
$$

Las variaciones volumétricas de expansión térmica se representan por la expresión.

$$
\begin{gathered}
V(T)=V(T o)\left[1+\alpha_{V}(T-T o)\right] \\
\alpha_{V} \approx 3 \alpha_{L}
\end{gathered}
$$

\section{Donde,}

$L(T), V(T)$ : longitud y volumen del cuerpo a temperatura $\mathrm{T},(\mathrm{m})$.

$L(T o), V(T o)$ : longitud y volumen del cuerpo a temperatura To, $(\mathrm{m})$.

$\alpha_{\mathrm{L}}$ : coeficiente lineal de expansión térmica, $\left({ }^{\circ} \mathrm{C}^{-1}\right)$.

$\alpha_{v}$ : coeficiente volumétrico de expansión térmica, $\left({ }^{\circ} \mathrm{C}^{-1}\right)$.

To: temperatura de referencia, $\left({ }^{\circ} \mathrm{C}\right)$.

$\mathrm{T}$ : temperatura del cuerpo, $\left({ }^{\circ} \mathrm{C}\right)$.

Para el vidrio Pyrex, el coeficiente lineal de expansión térmica para variaciones de temperatura menores de $100\left({ }^{\circ} \mathrm{C}\right)$, es $\mathrm{aL}=3.2 \times 10^{-6}\left({ }^{\circ} \mathrm{C}^{-1}\right)$, dato tomado de Wikipedia (10 Diciembre del 2017). Coeficiente de dilatación térmica.

\section{Datos}

Los datos experimentales de la densidad del aceite Shell Helix HX7 10W-40, fueron medidos empleando el siguiente material y equipo.

- Un litro de aceite Shell Helix HX7 10W-40.

- Un areómetro cuya temperatura de calibración fue 20 $\left({ }^{\circ} \mathrm{C}\right)$.

- Una hornilla eléctrica de Potencia máxima $=2500$ (W), 220 (VAC).

- Un recipiente cilíndrico de aluminio.

- Una probeta de laboratorio de vidrio, graduado, de $500(\mathrm{ml})$.

- Un termómetro de mercurio de $0-100\left({ }^{\circ} \mathrm{C}\right)$.

Figura 02: Medición de la densidad del aceite Shell Helix HX7 10W-40 en función de la temperatura

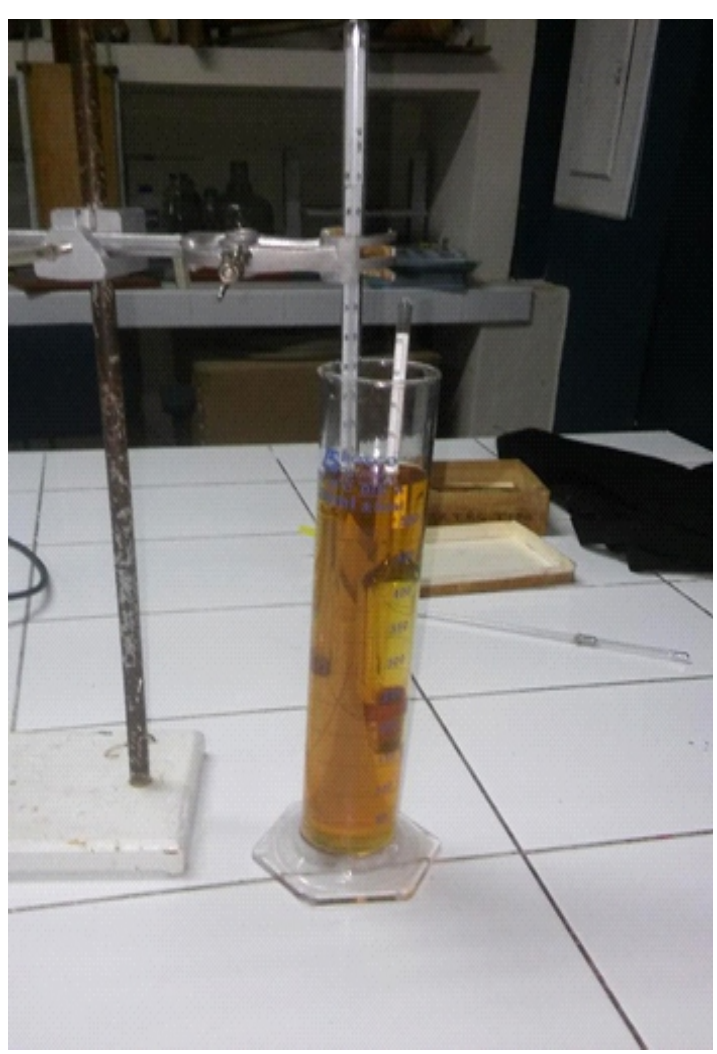

Fuente: Elaboración propia.

El procedimiento experimental fue el siguiente:

- El aceite lubricante se vertió en la probeta de laboratorio hasta alcanzar el nivel de $500(\mathrm{ml})$.

- El aceite se calentó en baño de maría hasta alcanzar la temperatura de $48.5\left({ }^{\circ} \mathrm{C}\right)$.

- La probeta con aceite se sacó del baño de maría y se dejó enfriar al medio ambiente.

- A medida que se enfriaba se tomaron datos de temperatura y densidad. Los datos de temperatura se tomaron con el bulbo del termómetro ubicado en el nivel $250(\mathrm{ml})$ de la probeta de laboratorio.

Los datos tomados directamente de los instrumentos, se presentan en la tabla 1. 